Research Paper

\title{
Validation of Urine-based Gene Classifiers for Detecting Bladder Cancer in a Chinese Study
}

\author{
Chengtao Han ${ }^{1,2^{*}}$, Lourdes Mengual $3^{*}$, Bin Kang 4,5 , Juan José Lozano ${ }^{6}$, Xiaoqun Yang7, Cuizhu Zhang ${ }^{1,2}$, , \\ Antonio Alcaraz ${ }^{3 凶}$, Ji Liang ${ }^{4,5}$ and Dingwei $\mathrm{Ye}^{1,2 \bowtie}$ \\ 1. Department of Urology, Fudan University Shanghai Cancer Center, Shanghai, P.R. China \\ 2. Department of Oncology, Shanghai Medical College, Fudan University, Shanghai, P.R. China \\ 3. Department and Laboratory of Urology, Hospital Clínic, Institut d'Investigacions Biomèdiques August Pi iSunyer, Universitat de Barcelona, Barcelona, \\ Spain \\ 4. Fudan University Shanghai Cancer Center - Institut Merieux Laboratory, Cancer Institute,Fudan University Shanghai Cancer Center, Shanghai, P.R. \\ China. \\ 5. bioMerieux (Shanghai) Company Limited, Shanghai, P.R. China \\ 6. CIBERehd. Plataforma de Bioinformática. IDIBAPS \\ 7. Department of Pathology, Rui Jin Hospital, School of Medicine, Shanghai Jiao-Tong University, Shanghai, P.R. China.
}

* Both authors have contributed equally to this work

$\square$ Corresponding and senior authors: Antonio Alcaraz, MD, Phone: +34-93-2275545; E-mail: AALCARAZ@clinic.cat, Ji Liang, PhD, Phone: +86-21-64438380; E-mail: jill.liang@biomerieux.com and Dingwei Ye, MD, Phone: +86-21-64175590; E-mail: dwyeli@163.com

(c) Ivyspring International Publisher. This is an open access article distributed under the terms of the Creative Commons Attribution (CC BY-NC) license (https://creativecommons.org/licenses/by-nc/4.0/). See http://ivyspring.com/terms for full terms and conditions.

Received: 2017.12.22; Accepted: 2018.04.15; Published: 2018.08.06

\begin{abstract}
Background: Current standard methods used to detect and monitor bladder cancer (BC) are invasive or have low sensitivity. We have previously reported in an international European study four non-invasive tests for BC diagnosis based on the gene expression patterns of urine.

Objective: to validate the tests in an independent Asian cohort.

Design, setting and participants: Prospective blinded study in which consecutive voided urine samples from BC patients and controls $(n=520)$ were collected in the Fudan University Shanghai Cancer Center from 2014-2016. Gene expression values were quantified using TaqMan Arrays. The same cut-off as previously reported for discrimination between tumours and controls was used in this validation study.

Results and limitations: Finally, a total of 257 tumour and 132 control urine samples were analysed. We found a high accuracy for the four gene classifiers in this independent Asian set, the classifiers composed of 5 and 10 genes achieved the best sensitivity $(80.54 \%$ and $81.32 \%$, respectively) maintaining a high specificity ( $91.67 \%$ and $85.61 \%$, respectively). Sensitivity of 5-gene (GS_D5) and 10-gene (GS_D10) expression classifiers in recurrent $\mathrm{BC}$ cases (78 and $79 \%$, respectively) is comparable to that of primary $\mathrm{BC}$ cases $(82 \%)$. Cytology and NMP22 identified $67 \%$ and $40 \%$, respectively, of tumours that have been diagnosed with our tests. In addition, influence of each studied gene was analyzed and showed similar gene rank between Chinese and Caucasian population.

Conclusions: Our study proves that our non-invasive diagnostic BC tests can be reproduced in independent cohorts and in an external laboratory. All the four gene classifiers have shown equal or superior performance to the current gold standard in the present and previously reported validation studies. Consequently, they may be taken for consideration as molecular tests applicable to clinical practice in the management of BC.
\end{abstract}

Patient summary: Our gene classifiers achieve sensitivities up to $90 \%$ in HR NMIBC and MIBC patients, while this achievement is comparatively lower in LR NMIBC ones.

Key words: Bladder cancer; Biomarkers; Gene expression; Gene classifiers; Non-invasive; Chinese

\section{Introduction}

As in other countries, urinary bladder cancer (BC) remains the second most frequent cause of mortality among genitourinary cancers in China, including approximately $4.8 / 10^{5}$ incidence and $2.2 / 10^{5}$ mortality rate in male in $2012[1,2]$. Although this two rates are not as high as those in western 
countries [3], the incidence and mortality rates of $\mathrm{BC}$ in China have increased gradually in the past few years [2].

The striking majority of malignant bladder tumours are urothelial cell carcinomas (UCC). Depending on the degree of tumour infiltration in the bladder wall, $\mathrm{BC}$ is classified as non-muscle invasive BC (NMIBC) accounting for $75 \%$ of tumours while the remainder are muscle invasive $\mathrm{BC}$ (MIBC) [4]. Although not typically life-threatening if detected early, NMIBC has up to $70 \%$ recurrence rate during the first two years after diagnosis, depending on the patient risk profile $[5,6]$. This recurrence phenomenon means that NMIBC patients may undergo up to 15 invasive procedures during the first 5 years of follow up, depending on the patients risk profile [7].

Current approaches for detecting both primary and recurrent disease rely on invasive cystoscopy aided by voided urine cytology. Cytology is a noninvasive technique and has high specificity $(90 \%$ to $96 \%$ ) [8], but lacks sensitivity especially in low risk tumours $(11 \%$ to $76 \%)$ [9]. Additionally, the inter-observer and intra-observer reproducibility of cytology is poor [10].

Invasive cystoscopy is associated with significant discomfort, possible infection and trauma. Moreover, cystoscopy misses up to $15 \%$ of the papillary and up to $30 \%$ of the flat lesions [11, 12]. In an effort to reduce the frequency of cystoscopies conducted, several noninvasive biomarkers such as nuclear matrix protein 22 (NMP22) test, have been approved by the Food and Drug Administration (FDA), albeit with performance rates remaining insufficient to replace or to guide current diagnostic methods [13]. Since the genetic nature of bladder tumours is heterogeneous, one possible reason of the lack performance of the assays is that they focus on a single or a limited number of biomarkers [14, 15]. In the last decade, several cancer-associated gene classifiers, obtained from voided urine, have been described with high diagnostic performances by different groups [14, 16-19]. Although the promising results presented, these multiple gene classifiers require large-scale prospective validations to prove its repeated efficiency and widespread application.

In this study, we have tested four gene expression classifiers previously developed and validated in Caucasian population [20], in an independent Asiatic cohort, to confirm its widespread clinical application. In addition, we have analyzed whether there is a difference in the influence of the genes included in the study to diagnostic performances between Chinese and Caucasian population.

\section{Materials and Methods}

\section{Clinical sampling and processing}

A total of 520 consecutive urine samples from patients with BC (336) and controls (184) were consecutively collected between September 2014 and March 2016 in the Fudan University Shanghai Cancer Center (FUSCC) and Shanghai $8^{\text {th }}$ people hospital after obtaining Institutional Review Board approval and patients' informed consents. Of the 520 urine samples collected, 96 samples (49 from the cancer and 47 from the control group) were excluded from study because they did not fulfill RNA quality criteria (a Cq value of GUSB $>23$; see materials and methods). Twenty-seven samples were excluded for incomplete and incorrect clinical information and eight samples were excluded for repeated testing (Figure 1). Thus, 389 urine specimens were finally analyzed, including 257 samples from patients treated with transurethral resection of the bladder (TURB) for primary or recurrent BC who had histologically confirmed tumours and 132 from controls with non-neoplastic urological disease (Table 1). Grade and stage of the tumours were determined according to WHO criteria [21] and the TNM classification [22], respectively. Tumours were classified according to their risk in 3 categories, including low risk NMIBC: Ta and T1 LG without associated CIS, high risk NMIBC: Ta or T1 LG with associated CIS, Ta or T1 HG, or Tis and MIBC: T2, T3 or T4 LG and HG with or without associated CIS. Voided urine samples (20 to $100 \mathrm{ml}$ ) were collected in sterile containers containing $4 \mathrm{ml} 0.5 \mathrm{M}$ EDTA ( $\mathrm{pH}$ 8.0). Urine samples were immediately stored at $4^{\circ} \mathrm{C}$ and processed within the next 24 hours. Samples were centrifuged at $1,000 \times \mathrm{g}$ for 10 minutes at $4^{\circ} \mathrm{C}$. Cell pellets were suspended in $1 \mathrm{ml}$ TRIzol reagent and frozen at $-80 \mathrm{C}$ until RNA extraction.

\section{Gene Expression Quantification}

RNA extraction, complementary DNA synthesis and gene expression quantification were performed in the FUSCC - Institut Merieux Laboratory as previously described [16, 17]. All the 16 target genes and two endogenous controls (GUSB and PPIA) analyzed in our previously reported studies were also analyzed in the present study [16]. Before gene expression quantification of the 16 target genes, an aliquot of $1 \mu \mathrm{l}$ of preamplified cDNA was applied to verify the actual amount of endogenous control GUSB by quantitative PCR (qPCR) and standard reaction and amplification conditions. Those samples that provide GUSB cycle quantification $(\mathrm{Cq})$ values lower than 18, were diluted with water to ensure a homogeneous amount of cDNA in all the samples and the correct quantification of mRNAs. Whereas those 
samples with a $\mathrm{Cq}$ value higher that 23 were excluded from the study. Real-time quantitative PCR data was processed with SDS 2.4. Previous defined gene thresholds [16] were used for all genes to record Cq values.

\section{Data Analysis}

Relative expression values (DCq) for the genes contained in the four evaluated predictive models (GS_D2, GS_D5, GS_D10 and GS_D12; Supplementary Table 1S) [13] were used to calculate the risk for the sample of presenting $\mathrm{BC}$. Raw data obtained from the qPCR platform was sent to Hospital Clinic (Barcelona, Spain) to be analized using a previously defined algorithm for each model which classify samples as tumours or controls All the researchers from the Hospital Clinic involved in this analysis of samples were blinded to the patients' clinical data, ensuring the reliability of the results. R-software was used for all calculations. Receiver Operating Characteristic (ROC) curves were generated using the Diagnosis Med (http:/ CRAN.Rproject.org/packageZDiagnosisMed) and pROC package [23]. Gene influence analysis was performed using R package globaltest [17].

\section{Results}

\section{Validation of Four Gene Expression classifiers}

A total of 389 urine samples were finally analyzed (Figure 1; Table 1). The performances of four diagnostic classifiers in the Chinese set are listed in Figure 2A. All the four gene classifiers achieved high diagnostic accuracy $(80.46 \%-84.32 \%$; AUC $=0.889$ 0.917). GS_D5 achieved the best diagnostic accuracy (84.32\%; AUC=0.911), with $80.54 \%$ SN and $91.67 \%$ SP. GS_D10 has the best SN (81.32\%), while GS_D12 has the best SP $(92.42 \%)$. Similarly to that of European studies, $\mathrm{SN}$ increased through the $\mathrm{BC}$ risk groups. It was lower in low risk NMIBC (54\%-58\%), while SN are up to $\sim 90 \%$ for high risk bladder cancer patients except for GS_D2 (85\%) (Figure 2B). Therefore, the study in the Chinese cohort has confirmed the performance results previously obtained in European Studies.

\section{Sensitivity in primary and recurrent BC cases}

Among the 257 cancer patients, 67 of them are recurrent and 190 are primary tumours. GS_D10 showed the best sensitivity, both in primary and recurrent cases (Figure 3A). Interestingly, the sensitivity of GS_D10 in recurrent cases (79\%) is comparable to that of primary tumours (82\%). Furthermore, sensitivities of GS_D10, which showed best sensitivity among the classifiers, in LR NMIBC, HR NMIBC and MIBC patients for primary and recurrent cases are shown in Figure 3B. There are no significant differences between primary and recurrent cases in all the comparisons (LR NMIBC: $\mathrm{P}=0.4233$; HR NMIBC: $\mathrm{P}=0.3459$; MIBC: $\mathrm{P}=0.2916)$.

Table 1. Clinical and histopathological variables for the patients and controls included in the study.

\begin{tabular}{lll}
\hline Variable & Tumor $\mathbf{( N = 2 5 7 )}$ & Control $\mathbf{( N = 1 3 2 )}$ \\
\hline Sex (\%) & $211(82.1)$ & $105(79.5)$ \\
Male & $46(17.9)$ & $27(20.5)$ \\
Female & & \\
Age (yr) & 62.1 & 63.6 \\
Mean & $24-89$ & $29-90$ \\
Range & & \\
Grade & 72 & \\
NMIBC LR & 146 & \\
NMIBC HR & 39 & \\
MIBC & & \\
Urological condition & & 10 \\
Normal & & 73 \\
BPH & & 11 \\
Urinary tract infection & & 27 \\
Calculus & & 11 \\
Others & & \\
\hline
\end{tabular}

Table 2. Sensitivity comparison of 4 gene classifiers and cytology

\begin{tabular}{lllll}
\hline Grade & Overall & LR & HR & MIBC \\
\hline N & $154 / 257$ & 42 & 89 & 23 \\
cytology & $55 \%$ & $19 \%$ & $67 \%$ & $74 \%$ \\
GS_D2 & $78 \%$ & $57 \%$ & $87 \%$ & $83 \%$ \\
GS_D5 & $81 \%$ & $60 \%$ & $90 \%$ & $87 \%$ \\
GS_D10 & $82 \%$ & $60 \%$ & $91 \%$ & $87 \%$ \\
GS_D12 & $79 \%$ & $57 \%$ & $88 \%$ & $83 \%$ \\
\hline
\end{tabular}

\section{Cytology and NMP22 results BC samples}

Cytology results were available for 154 (60\%) of the 257 BC patients included in the study. We compared sensitivity of four gene classifiers to those of cytology (Table 2). In this subset of patients, overall sensitivity of cytology was $55 \%$, much lower than that of the four gene classifiers (78\%-82\%). $\mathrm{SN}$ of the cytology in LR NMIBC, HR NMIBC and MIBC patients was $19 \%, 67 \%$ and $74 \%$ respectively, lower of those sensitivities of four gene models (Table 2). Further comparison analysis of 5-gene classifier (GS_D5) and cytology results showed that all positive cytologies were confirmed by the GS_D5 except in two cases $(2 \%)$. On the contrary, of all the patients diagnosed GS_D5, cytology only detected BC in $66 \%$ of them (Figure 4A).

NMP22 test have been done for $109(42 \%)$ of the 257 BC patients included in our study. Overall, sensitivity of NMP22 test in this subset of patients was $38 \%$ while $\mathrm{SN}$ of the gene classifiers ranged from $74 \%$ to $88 \%$. SN of the NMP22 in LR NMIBC, HR NMIBC and MIBC patients was $21 \%, 45 \%$ and $42 \%$ respectively, more than half lower of those sensitivities of four gene models (Table 3). Furthermore, GS_D5 detects BC in 34 (83\%) of all 41 
positive NMP22 cases. On the contrary, of all the patients diagnosed by GS_D5, NMP22 tests only detect $\mathrm{BC}$ in $40 \%$ of them (Figure $4 \mathrm{~B}$ ).

Table 3. Sensitivity comparison of 4 gene classifiers and NMP22 tests

\begin{tabular}{lllll}
\hline & Overall & LR & HR & MIBC \\
\hline N & $109 / 257$ & 29 & 60 & 19 \\
NMP22 & $38 \%$ & $21 \%$ & $45 \%$ & $40 \%$ \\
GS_D2 & $76 \%$ & $48 \%$ & $88 \%$ & $80 \%$ \\
GS_D5 & $78 \%$ & $55 \%$ & $86 \%$ & $87 \%$ \\
GS_D10 & $74 \%$ & $45 \%$ & $86 \%$ & $80 \%$ \\
GS_D12 & $76 \%$ & $52 \%$ & $85 \%$ & $87 \%$ \\
\hline
\end{tabular}

\section{Influence of each studied gene in Chinese population}

IGF2, SLC1A6 and CRH are the most influential genes in both Caucasian and Chinese populations. All the high influent genes included in the top 8 genes are almost the same; except for MAGEA3, that has more influence in the European cohort, while ANXA10 ranks higher in the Chinese validation set (Figure 4D). Those data were consistent with the good performances presented by the four gene classifiers on the Chinese validation cohort.

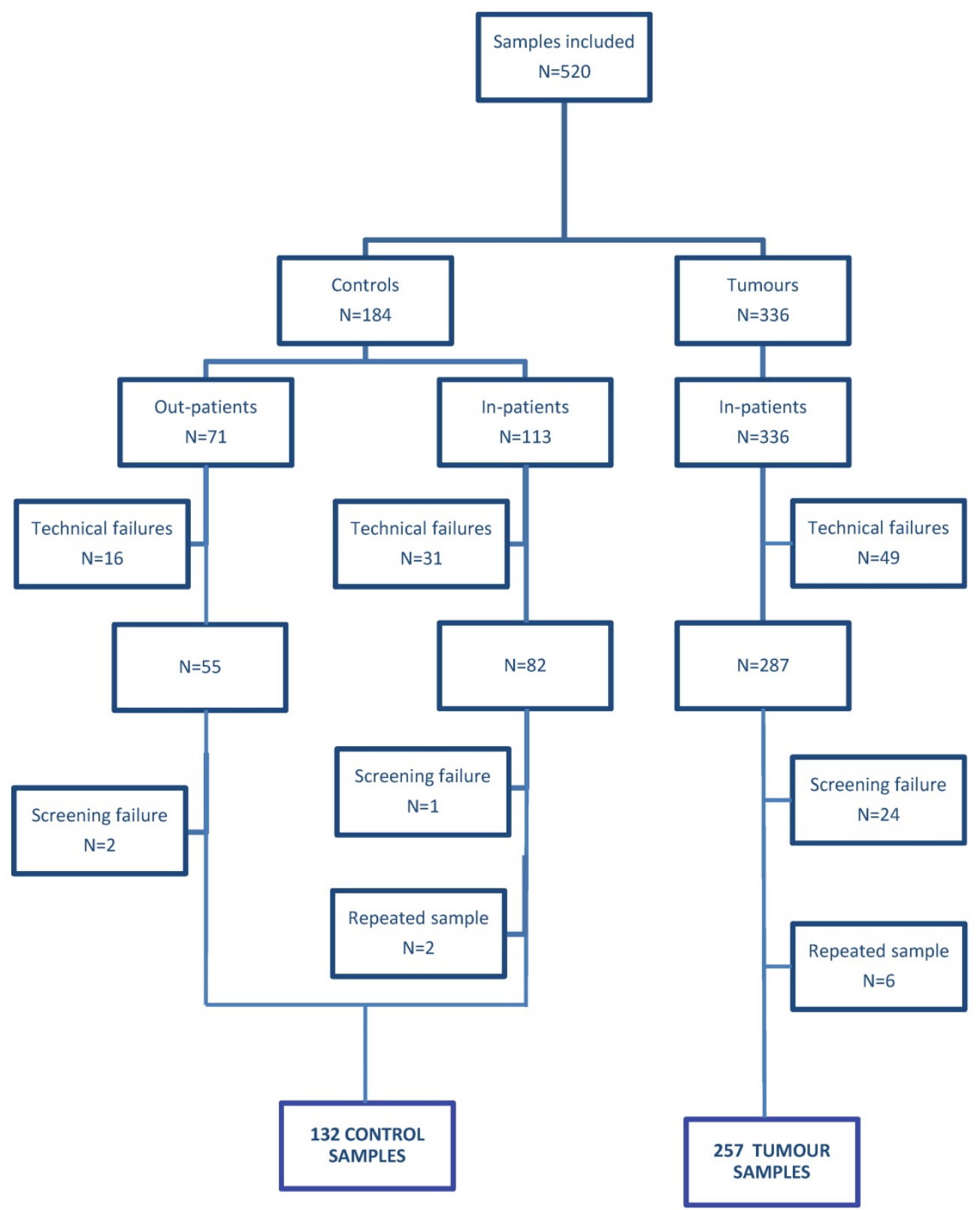

Figure 1: Flow diagram of participants satisfying the criteria for inclusion. Technical failure: samples that yielded insufficient RNA and samples that did not meet the GUSB RNA quality control; see material and methods. In-patients: patients samples collected in FUSCC; Out-patients: samples collected in Shanghai $8^{\text {th }}$ people hospital. 
A

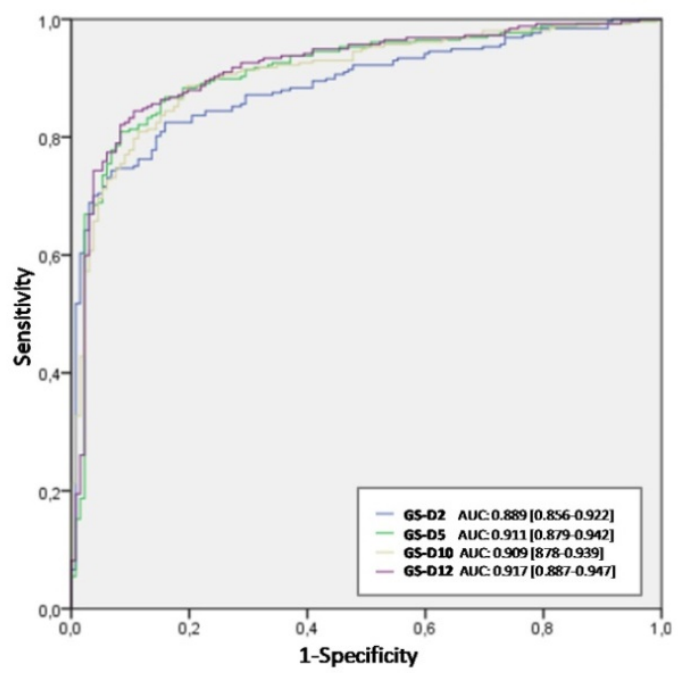

\begin{tabular}{cccccc}
\hline Models & Accuracy & Sensitivity & Specificity & PPV & NPV \\
\hline GS_D2 & $80.46 \%$ & $76.26 \%$ & $88.64 \%$ & $92.89 \%$ & $65.73 \%$ \\
GS_D5 & $84.32 \%$ & $80.54 \%$ & $91.67 \%$ & $94.95 \%$ & $70.76 \%$ \\
GS_D10 & $82.78 \%$ & $81.32 \%$ & $85.61 \%$ & $91.67 \%$ & $70.19 \%$ \\
GS_D12 & $83.29 \%$ & $78.60 \%$ & $92.42 \%$ & $95.28 \%$ & $68.93 \%$ \\
\hline
\end{tabular}

B

\section{Prediction Sensitivity for different models in different patients}

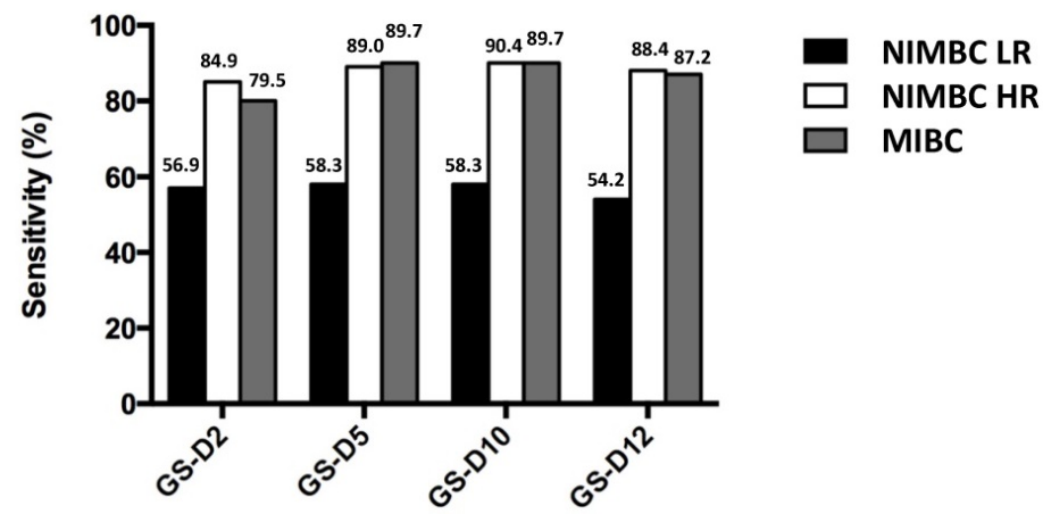

Figure 2: Diagnostic performance of 4 gene expression classifiers in the Chinese validation cohort. A) ROC curves and overall diagnostic performances of the 4 diagnostic gene expression classifiers in the Chinese cohort. B) SN of 4 gene expression classifiers in BC risk groups. Abbreviation: AUC, area under curve. PPV, positive predictive value. NPV, negative predictive value

\section{Discussion}

Currently, cystoscopy is considered the gold standard method to diagnose and monitor BC, but misses up to $15 \%$ of the papillary and up to $30 \%$ of the flat recurrences [11, 12]. Furthermore, cystoscopy is expensive, invasive and bothersome to patients. Urine cytology, on the other hand, has a high specificity ( $\mathrm{SP}=96 \%)$, but lacks sensitivity $(\mathrm{SN}=44 \%)$ especially in low risk tumours [24]. Additionally, the interobserver and intraobserver reproducibility of cytology is poor
[10]. The combination of both techniques achieves a high SN and SP in the diagnosis and monitoring of the disease (SN: 71\%, SP: 96\%) [25]. Nevertheless, the invasiveness of cystoscopy has led to the search for biomarkers in urine. This is especially important in the surveillance of $\mathrm{BC}$ patients. The high recurrence rate of $\mathrm{BC}$ leads to a life-time surveillance with frequent invasive procedures which are associated to significant pain, anxiety and financial cost to the BC patients. Our current and previous studies demonstrated that the non-invasive urine biomarkers 
A

\begin{tabular}{ccc}
\hline & Primary BC & Recurrent BC \\
\hline N & 190 & 67 \\
GS_D2 & $80 \%$ & $67 \%$ \\
GS_D5 & $82 \%$ & $78 \%$ \\
GS_D10 & $82 \%$ & $79 \%$ \\
GS_D12 & $81 \%$ & $73 \%$ \\
\hline
\end{tabular}

B

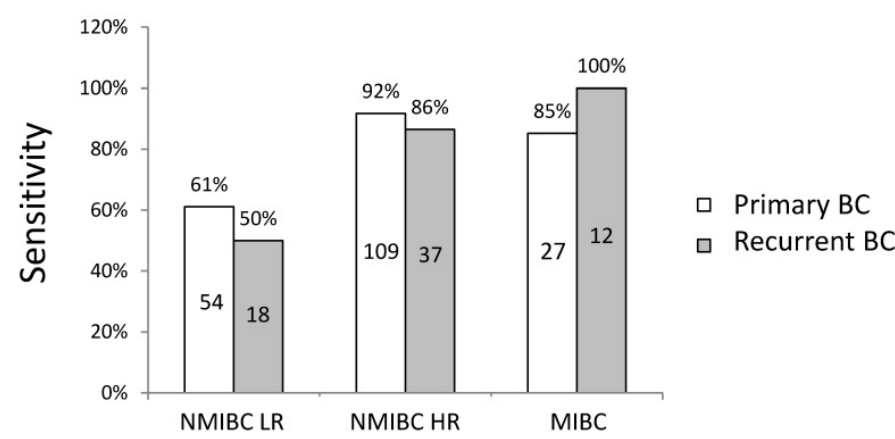

Figure 3: Diagnostic performance of 4 the gene expression classifiers in primary and recurrent BC. A) SN of 4 gene expression classifiers in primary and recurrent BC. B) SN of the 10-gene expression classifier (GS_D10) between primary and recurrent cases in BC risk groups. Numbers in the boxes indicate the patient numbers for each group.

tests achieved accuracy values (SN up to $80 \%$ and SP up to $90 \%$ ) for $\mathrm{BC}$ diagnosis in the range of that achieved for the gold standard. Furthermore, the good diagnostic performances were observed not only in BC patients with primary tumours but also on recurrent cases. Therefore, urine biomarkers tests could be a potential tool that aid to reduce the frequency of cystoscopies in selected patients.

A number of molecular tests have been developed to achieve this goal. Some of these tests include NMP-22 [25-28] bladder tumour antigen (BTA) [29], Survivin [30], RNA [19, 31] or MicroRNA profiling [32] and fluorescence in situ hybridization analysis for chromosomal abnormalities [9]. However, most of the biomarkers reported above have limited sensitivity, thus by themselves, have not proven to be accurate enough to replace cystoscopy or even cytology. In a meta-analysis of 57 studies none of them achieved a SN $>69 \%$, except $78 \%$ SN for ImmunoCyt (Scimedx, SP: 78\%), with an overall moderate SP ranged of $74 \% \sim 88 \%$ [9]. Consistently, in our study, the diagnostic performance of NMP22 (BladderChek, Alere) test is not sufficient to skip one cystoscopy resulting in a safe approach. In contrast to the moderate performance of one singe marker [33, 34], multiplex biomarkers panel usually show better performances [12-16, 18, 20, 31, 35, 36]. Urquidi et al reported a 14-gene panel in 2012 with 90\% SE and
$100 \%$ SP for BC diagnosis, although more high grade patients included in the cohort [19]. Recently, a DNA methylation signature (A 150 CpG loci biomarker panel) identified by high-throughput DNA sequencing showed a perfect performance ( $98 \%$ SE and $97 \%$ SP) on primary BC detection [37]. However, for routine clinical diagnostic use of those promising panels, they need to be tested further and prospective validated in heterogeneous patient populations. So far, our method is the first test validated in both Caucasian and Chinese populations.

Similar to previous results in European studies, the four gene classifiers showed comparatively lower SN (54 to 58\%) in LR NMIBC patients than those of HR ones (80 to $90 \%)$. Actually, 30 of the 46 misclassified tumour samples by GS_D5, one of the best performer in all series, are LR NMIBC. However, the SN of four gene classifiers in LR NMIBC is still $40 \%$ higher than that of cytology and 30\% higher than that of NMP22 tests. On the other hand, considering high recurrence rate and life threaten of HR NMIBC, the performance of the tests in this subgroup of tumours is of great importance. Our gene classifiers achieve sensitivities up to $90 \%$ in HR NMIBC and MIBC patients, that is $10-20 \%$ higher than those of cytology and $40-50 \%$ higher than those of NMP22 test in the present cohort. One limitation of our study is the lack of cytology and NMP22 test in the control group that does not allow for a direct calculation of the SP of cytology and NMP22 test in our own series. But total $\mathrm{SP}$ of our gene classifiers are in the range as that of reported for cytology [7].

Another limitation of the current study is that the prevalence of tumours in this cohort is higher than the disease prevalence in urologic practice [7], so evaluation of the validation study cohort is likely to provide an overly optimistic assessment of the positive predictive value (PPV) and an overly pessimistic assessment of the negative predictive value (NPV). For an estimated real prevalence of $10 \%$ in urologic practice, NPV would be above $90 \%$ for all gene classifiers.

Although these gene classifiers have been already validated in patients from multiple centers, this was the first time they were tested outside Europe. Thus, we wondered if there is any heterogeneity of $\mathrm{BC}$ gene expression between the two populations that could affect the performance of the gene classifiers. Therefore, we analyzed the influence of each gene included in the four classifiers (Figure 4C). Surprisingly, ranks of the gene influences on the 
A

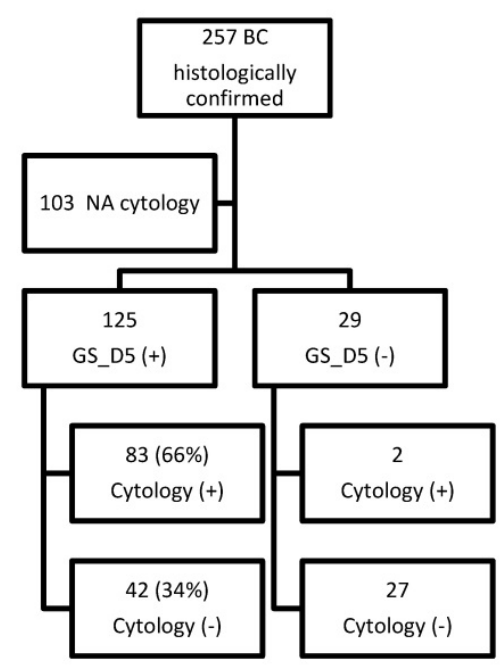

B

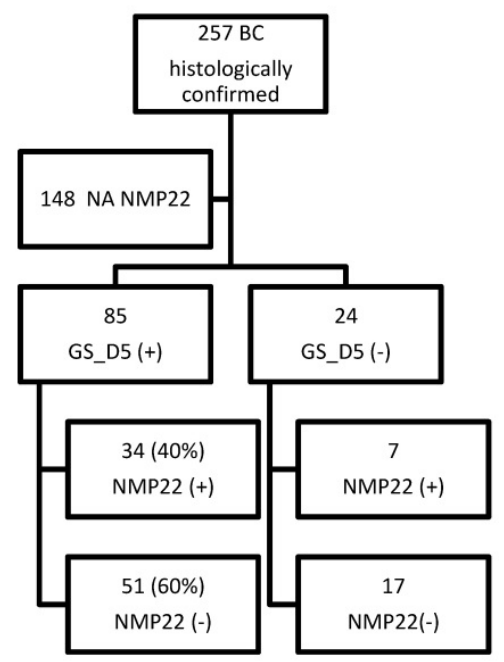

C

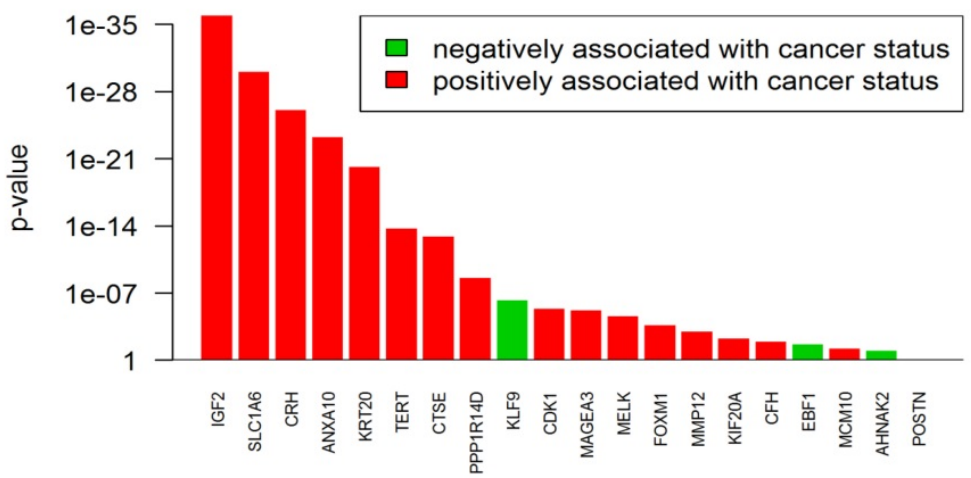

$\mathrm{D}$

\begin{tabular}{ccc}
\hline Rank & European & Chinese \\
$\mathbf{1}$ & IGF2 & IGF2 \\
$\mathbf{2}$ & CRH & SLC1A6 \\
$\mathbf{3}$ & SLC1A6 & CRH \\
$\mathbf{4}$ & KRT20 & ANXA10 \\
$\mathbf{5}$ & CTSE & KRT20 \\
$\mathbf{6}$ & MAGEA3 & TERT \\
$\mathbf{7}$ & PPP1R14D & CTSE \\
$\mathbf{8}$ & TERT & PPP1R14D \\
\hline
\end{tabular}

Figure 4: Performance comparison of the 4 gene expression classifiers with cytology and NMP22 results. Comparison of the 5-gene classifier (GS_D5) results with cytology (A) and NMP22 results (B). C) Influence on BC diagnosis of each studied gene in the Chinese validation set. D) Comparison of influence top-ranked genes between Caucasian and Chinese populations.

BC diagnosis were very similar between Caucasian and Chinese population, although there are minor variations on the priority of those genes to predict $B C$ between the two populations. We-found that MAGEA3, one of the two genes included in GS_D2, seems to be more important in Caucasian population, while ANXA10 shows higher influence in Chinese population. This could possibly explain why when

comparing to the GS_D5, GS_D10 and GS_D12 models, GS_D2 showed comparatively lower $\mathrm{SN}$ $(75.49 \%)$ in the Chinese cohort. This minor discrepancy could be affected by the different enrolment of the two cohorts, such as BC grades, primary or recurrent cases distribution, or it could be explained by the heterogeneity of $B C$ gene expression between the two populations. Further studies of the classifiers on Asiatic cohorts may be provide more evidence about it.

Taken together, this blinded and independent study proves that our non-invasive diagnostic BC tests can be reproduced in the Chinese cohort and in an external laboratory. All the four gene expression classifiers have shown equal or superior performance to the current gold standard in the present and previously reported validation studies. Consequently, they may be taken for consideration as a molecular test applicable to clinical practice in the management of $\mathrm{BC}$.

\section{Supplementary Material}

Supplementary table.

http://www.jcancer.org/v09p320 8s1.pdf

\section{Acknowledgements}

This work was supported by bioMerieux, Fina Biotech and Biofina Diagnostics. We thank all the patients who participated in this study and all the staff and nurses from the Urology Department of the Fudan University Shanghai Cancer Center for collaborating in collecting urine samples.

\section{Competing Interests}

Lourdes Mengual, Juan José Lozano, and Antonio Alcaraz are inventors of patents WO 2008/113870 and WO 2014/118334 applied by Fina Biotech S.L. All other authors declared no conflicts of interest. 


\section{References}

[1] Chen W, Zheng R, Zuo T, Zeng H, Zhang S, He J. National cancer incidence and mortality in China, 2012. Chinese journal of cancer research $=$ Chung-kuo yen cheng yen chiu 2016;28:1-11.

[2] Pang C, Guan Y, Li H, Chen W, Zhu G. Urologic cancer in China. Japanese journal of clinical oncology 2016;46:497-501.

[3] Ferlay J, Soerjomataram I, Dikshit R, Eser S, Mathers C, Rebelo M, et al. Cancer incidence and mortality worldwide: sources, methods and major patterns in GLOBOCAN 2012. International journal of cancer 2015;136:E359-86.

[4] Nielsen ME, Smith AB, Meyer AM, Kuo TM, Tyree S, Kim WY, et al. Trends in stage-specific incidence rates for urothelial carcinoma of the bladder in the United States: 1988 to 2006. Cancer 2014;120:86-95.

[5] Babjuk M, Burger M, Zigeuner R, Shariat SF, van Rhijn BW, Comperat E, et al. EAU guidelines on non-muscle-invasive urothelial carcinoma of the bladder: update 2013. European urology 2013;64:639-53.

[6] Donat SM. Evaluation and follow-up strategies for superficial bladder cancer. The Urologic clinics of North America 2003;30:765-76.

[7] Babjuk M, Bohle A, Burger M, Capoun O, Cohen D, Comperat EM, Hernandez V, Kaasinen E, Palou J, Roupret M, van Rhijn BW, Shariat SF, Soukup V, et al. EAU Guidelines on Non-Muscle-invasive Urothelial Carcinoma of the Bladder: Update 2016. Eur Urol. 2016

[8] Glas AS, Roos D, Deutekom M, Zwinderman AH, Bossuyt PM, Kurth KH, Tumor markers in the diagnosis of primary bladder cancer. A systematic review. The Journal of urology 2003;169:1975-82.

[9] Lokeshwar VB, Habuchi T, Grossman HB, Murphy WM, Hautmann SH, Hemstreet GP, 3rd, et al. Bladder tumor markers beyond cytology: International Consensus Panel on bladder tumor markers. Urology 2005;66:35-63.

[10] Sherman AB, Koss LG, Adams SE. Interobserver and intraobserver differences in the diagnosis of urothelial cells. Comparison with classification by computer. Anal Quant Cytol. 1984; 6: 112-120

[11] Grossman HB, Gomella L, Fradet Y, Morales A, Presti J, Ritenour C, Nseyo U, Droller MJ, PC 302/01 Study Group. A phase III, multicenter comparison of hexaminolevulinate fluorescence cystoscopy and white light cystoscopy for the detection of superficial papillary lesions in patients with bladder cancer. J Urol. 2007; 178: 62-67.

[12] Fradet Y, Grossman HB, Gomella L, Lerner S, Cookson M, Albala D, Droller MJ. A comparison of hexaminolevulinate fluorescence cystoscopy and white light cystoscopy for the detection of carcinoma in situ in patients with bladder cancer: a phase III, multicenter study. J Urol. 2007; 178: 68-73.

[13] Chou R, Gore JL, Buckley D, Fu R, Gustafson K, Griffin JC, et al. Urinary Biomarkers for Diagnosis of Bladder Cancer: A Systematic Review and Meta-analysis. Annals of internal medicine 2015;163:922-31.

[14] Holyoake A, O'Sullivan P, Pollock R, Best T, Watanabe J, Kajita Y, et al. Development of a multiplex RNA urine test for the detection and stratification of transitional cell carcinoma of the bladder. Clinical cancer research : an official journal of the American Association for Cancer Research 2008;14:742-9.

[15] Xie XY, Yang X, Zhang JH, Liu ZJ. Analysis of hTERT expression in exfoliated cells from patients with bladder transitional cell carcinomas using SYBR green real-time fluorescence quantitative PCR. Annals of clinical biochemistry 2007;44:523-8

[16] Mengual L, Burset M, Ribal MJ, Ars E, Marin-Aguilera M, Fernandez M, et al. Gene expression signature in urine for diagnosing and assessing aggressiveness of bladder urothelial carcinoma. Clinical cancer research : an official journal of the American Association for Cancer Research 2010;16:2624-33

[17] Mengual L, Ribal MJ, Lozano JJ, Ingelmo-Torres M, Burset M, Fernandez PL, et al. Validation study of a noninvasive urine test for diagnosis and prognosis assessment of bladder cancer: evidence for improved models. The Journal of urology 2014;191:261-9.

[18] O'Sullivan P, Sharples K, Dalphin M, Davidson P, Gilling P, Cambridge L, et al. A multigene urine test for the detection and stratification of bladder cancer in patients presenting with hematuria. The Journal of urology 2012;188:741-7.

[19] Urquidi V, Goodison S, Cai Y, Sun Y, Rosser CJ. A candidate molecular biomarker panel for the detection of bladder cancer. Cancer epidemiology, biomarkers \& prevention : a publication of the American Association for Cancer Research, cosponsored by the American Society of Preventive Oncology 2012;21:2149-58.

[20] Ribal MJ, Mengual L, Lozano JJ, Ingelmo-Torres M, Palou J, Rodriguez-Faba $\mathrm{O}$, et al. Gene expression test for the non-invasive diagnosis of bladder cancer: A prospective, blinded, international and multicenter validation study. European journal of cancer 2016;54:131-8.

[21] Lopez-Beltran A, Sauter G, Gasser T, et al. Tumours of the urinary system. In: Eble JN, Sauter G, Epstein JI, Sesterhenn IA, editors. Pathology and genetics of tumours of the urinary system and male genital organs. World Health Organization classification of tumours. Lyon: IARC Press; 2004.

[22] Sobin LH, Wittekind CH. TNM classification of malignant tumours. International Union Against Cancer. 6th ed. New York: Jonh Wiley \& Sons; 2002.

[23] Robin X, Turck N, Hainard A, Tiberti N, Lisacek F, Sanchez JC, et al. pROC: an open-source package for R and $\mathrm{S}+$ to analyze and compare ROC curves. BMC bioinformatics 2011;12:77.

[24] Brown FM. Urine cytology. It is still the gold standard for screening? Urol Clin North Am 2000;27:25-37.
[25] Mowatt G, Zhu S, Kilonzo M, Boachie C, Fraser C, Griffiths TR, et al. Systematic review of the clinical effectiveness and cost-effectiveness of photodynamic diagnosis and urine biomarkers (FISH, ImmunoCyt, NMP22) and cytology for the detection and follow-up of bladder cancer. Health technology assessment 2010;14:1-331, iii-iv.

[26] Shariat SF, Marberger MJ, Lotan Y, Sanchez-Carbayo M, Zippe C, Ludecke G, et al. Variability in the performance of nuclear matrix protein 22 for the detection of bladder cancer. The Journal of urology 2006;176:919-26; discussion 26.

[27] Chang $\mathrm{YH}$, Wu CH, Lee YL, Huang PH, Kao YL, Shiau MY. Evaluation of nuclear matrix protein-22 as a clinical diagnostic marker for bladder cancer. Urology 2004;64:687-92.

[28] Pichler R, Tulchiner G, Fritz J, Schaefer G, Horninger W, Heidegger I. Urinary UBC Rapid and NMP22 Test for Bladder Cancer Surveillance in Comparison to Urinary Cytology: Results from a Prospective Single-Center Study. I.Int J Med Sci. 2017;14(9):811-819.

[29] D'Costa JJ, Goldsmith JC, Wilson JS, Bryan RT, Ward DG. A Systematic Review of the Diagnostic and Prognostic Value of Urinary Protein Biomarkers in Urothelial Bladder Cancer. Bladder Cancer. 2016;2(3):301-317.

[30] Chang Y, Xu J, Zhang Q. Microplate magnetic chemiluminescence immunoassay for detecting urinary survivin in bladder cancer. Oncol Lett. 2017;14(4):4043-4052.

[31] Urquidi V, Netherton M, Gomes-Giacoia E, Serie D, Eckel-Passow J, Rosser CJ, et al. Urinary mRNA biomarker panel for the detection of urothelial carcinoma. Oncotarget 2016;7(25):38731-38740

[32] Sapre N, Macintyre G, Clarkson M, Naeem H, Cmero M, Kowalczyk A, et al. A urinary microRNA signature can predict the presence of bladder urothelial carcinoma in patients undergoing surveillance. Br J Cancer. 2016 Feb 16; 114(4): 454-462. Published online 2016 Jan 26. doi: 10.1038/bjc.2015.472

[33] Tilki D, Burger M, Dalbagni G, Grossman HB, Hakenberg OW, Palou J, et al. Urine markers for detection and surveillance of non-muscle-invasive bladder cancer. European urology 2011;60:484-92.

[34] Xylinas E, Kluth LA, Rieken M, Karakiewicz PI, Lotan Y, Shariat SF. Urine markers for detection and surveillance of bladder cancer. Urologic oncology 2014;32:222-9.

[35] Frantzi M, van Kessel KE, Zwarthoff EC, Marquez M, Rava M, Malats N, et al. Development and Validation of Urine-based Peptide Biomarker Panels for Detecting Bladder Cancer in a Multi-center Study. Clinical cancer research : an official journal of the American Association for Cancer Research 2016;22:4077-86.

[36] Chen LM, Chang M, Dai YF, Chai KX, Dyrskjøt L, Sanchez-Carbayo M, et al. External Validation of a Multiplex Urinary Protein Panel for the Detection of Bladder Cancer in a Multicenter Cohort. Cancer Epidemiol Biomarkers Prev. 2014;23(9): 1804-1812.

[37] Feber A, Dhami P, Dong LQ, Winter P, Tan WS, Martínez-Fernández M, et al. UroMark-a urinary biomarker assay for the detection of bladder cancer. Clin Epigenetics. 2017; 9:8. 